\title{
Validation Study of SNPs in CAPN1-CAST Genes on the Tenderness of Muscles (Longissimus thoracis and Semimembranosus) in Hanwoo (Korean Cattle)
}

\author{
Hyo Jun Lee ${ }^{1,+}$, Shil Jin ${ }^{1,+}$, Hyoun-Joo Kim ${ }^{1,2}$, Mohammad Shamsul Alam Bhuiyan ${ }^{3}{ }^{\circ}$, \\ Doo Ho Lee ${ }^{1}$, Soo Hyun Lee ${ }^{1}$, Sung Bong Jang ${ }^{1}{ }^{\mathbb{D}}$, Man Hye Han ${ }^{2}$ and Seung Hwan Lee ${ }^{1, *}$ \\ 1 Division of Animal and Dairy Science, Chungnam National University, Daejeon 305-764, Korea; \\ gywns6298@naver.com (H.J.L.); wlstlf21@cnu.ac.kr (S.J.); joos0621@korea.kr (H.-J.K.); \\ stonecold@daum.net (D.H.L.); lhyungm@cnu.ac.kr (S.H.L.); jsbng8615@gmail.com (S.B.J.) \\ 2 Hanwoo Research Institute, National Institute of Animal Science, PyeongChang 232-956, Korea; \\ hanmhh@korea.kr \\ 3 Department of Animal Breeding and Genetics, Bangladesh Agricultural University, \\ Mymensingh 2202, Bangladesh; msabhuiyan@gmail.com \\ * Correspondence: slee46@cnu.ac.kr; Tel.: +82-42-821-5772; Fax: +82-42-825-9754 \\ + Hyo Jun Lee and Shil Jin contributed equally to this work.
}

Received: 13 August 2019; Accepted: 11 September 2019; Published: 17 September 2019

check for updates

Simple Summary: For meat tenderness, single nucleotide polymorphisms (SNPs) in the $\mu$-calpain (CAPN1) and calpastatin (CAST) genes have been reported to be associated with Warner-Bratzler shear force (WBSF) in different cattle populations, including Korean Hanwoo cattle. In this study, we validated the association of seven SNPs in CAPN1 and CAST genes with meat tenderness in two different muscle cuts tenderness in the Longissimus thoracis (LT) and Semimembranosus (SM) muscles. Two SNPs in CAPN1 and one SNPs in CAST genes showed association with WBSF of both muscle types. Furthermore, of twelve reconstructed haplotypes, six demonstrated significant associations with WBSF values. These findings may be one of the strong evidences that CAPN1 and CAST gene mutations are strongly associated with WBSF. The information of significantly-associated SNPs and the resulted haplotypes could be utilized in the Hanwoo breeding program for further genetic improvement of tenderness traits.

\begin{abstract}
Previous studies demonstrated that polymorphisms in the $\mu$-calpain (CAPN1) and calpastatin (CAST) genes had significant effects on meat tenderness in different cattle populations. The aim of this study was to validate the potential association of seven single nucleotide polymorphisms (SNPs) harbored in these two candidate genes with meat tenderness in the Longissimus thoracis (LT) and Semimembranosus (SM) muscles. A total of 1000 animals were genotyped using TaqMan SNP genotyping arrays, and the meat tenderness of two muscle (LT and SM at 7 days post-slaughter) was assessed based on Warner-Bratzler WBSF (WBSF) testing. We observed significant associations of the CAPN1:c.580T>C, CAPN1:c.658T >C and CAST:c.1985G >C polymorphisms $(p<0.05)$ with the WBSF values in the LT and SM muscles. Additive effects of the $C$ allele in CAPN1:c.580T $>C$ and CAST:c.1985G $>C$ were associated with an increase of 0.16 and $0.15 \mathrm{~kg}$, and 0.08 and $0.26 \mathrm{~kg}$ WBSF in the LT and SM, respectively; CAPN1:c.658T>C had negative effects on the WBSFs. Furthermore, six reconstructed haplotypes demonstrated significant associations with WBSF values $(p<0.05)$. In conclusion, the significant associations identified between the SNPs in CAPN1, CAST and WBSF values could be utilized in marker-assisted selection programs in order to improve the beef tenderness of Hanwoo cattle.
\end{abstract}

Keywords: single nucleotide polymorphism; $\mu$-calpain; calpastatin; muscle; Hanwoo 


\section{Introduction}

Tenderness is an important meat quality determinant in the beef industry, as consumers are willing to pay more for lean and tender meat [1]. Meat tenderness, the most highly researched palatability characteristic, is influenced by multiple factors, including marbling, juiciness, and flavor [2]. Compared to past trends, Korean consumers currently prefer highly marbled meat and exhibit a strong willingness to purchase tender meat that promotes the utilization of a system that ensures high-quality meat production [3]. To identify reliable gene markers that explain the large genetic and phenotypic variations in beef tenderness, an association analysis is required to validate previously tested gene markers and their subsequent effects in a large independent reference population [4]. For meat tenderness, SNPs in the CAPN1 and CAST genes have been reported to be associated with WBSF in several cattle breeds [5-8].

CAPN1/CAST is an endogenous calcium-dependent proteinase system that mediates the proteolysis of key myofibrillar proteins during the postmortem storage of carcasses and cuts of meat at refrigerated temperatures. CAPN1 is responsible for the breakdown of myofibrillar proteins and is associated with meat tenderness [9]. CAST inhibits $\mu$ - and m-calpain activity, which regulates postmortem proteolysis. The effects of DNA markers on these genes have been validated in many temperate breeds, tropical-adapted zebu, and tropical composites [4,10-12]. Genetic tests for meat tenderness that utilize SNPs in CAST and CAPN1 have already been commercialized by Igenity TenderGENE (Merial Ltd., Atlanta, GA, USA) and GeneSTAR Elite Tender (Genetic Solutions Pty. Ltd., Brisbane, Australia). In addition, seven SNPs in CAPN1-CAST systems affecting meat tenderness were also identified in Hanwoo cattle [13]. However, the resulting effects of these gene markers need to be validated in a large reference population in order to commercialize a palatability prediction system. In this study, we validated the association of seven SNPs in CAPN1 and CAST genes with meat tenderness in two different muscle cuts (LT and SM) in Hanwoo steers.

\section{Materials and Methods}

\subsection{Animal and Phenotype Measurement}

To validate CAPN1-CAST SNP effect, the DNA and Phenotypes datasets $(\mathrm{n}=1000)$ was derived from samples collected from the Daegwallyeong Hanwoo Co. abattoir (Gangwon province, Korea). Animal Care and Use Committee approval was obtained for this study based on instructions for animal health and welfare outlined by the National Institute of Animal Science, Rural Development Administration (RDA), Korea. Management and feeding system were similar for the studied population. Calves weaned at 5 or 6 month and individuals were transferred by 10 per each pen. All calves were freely fed with a growing ration that consisted of hay and concentrate mixtures from 6 to 11 month of age. The percentage of TDN and CP were $70 \%$ and $15 \%$, respectively. After that, steers were fed differently between early fattening period (12-15 mo), middle fattening period (16-21 mo) and final fattening period (22-29 mo). Individuals received concentrates and a rice straw-based ration with proportion in total feed of 2.5:1, 4.5:1 and 8:1 for each fattening period. Percentages of CP and TDN of the concentrates were $14 \%$ and $71 \%, 13 \%$ and $72 \%$ and $12 \%$ and $73 \%$, respectively. All steers were slaughtered at 30 months of age and muscle samples $(1.5 \mathrm{~kg})$ were collected from the Longissimus thoracis (LT) and Semimembranosus (SM) muscles to measure Warner-Bratzler WBSF (WBSF) values. This half sib steer population $(n=1000)$ was derived from 120 progeny tested bulls and unrelated dams (2-10 progeny per bull). The WBSF values of two cooked steaks (LT and SM at 7 days post-slaughter) was measured according to the method of Wheeler et al. [14]. Steaks of $2.5 \mathrm{~cm}$ with an $\sim 80 \mathrm{~g}$ weight were put into polyethylene bags. The bags were heated in a water bath at $80^{\circ} \mathrm{C}$ for 30 min until the internal temperature of the steak reached $70{ }^{\circ} \mathrm{C}$. After cooking, the meat samples were gradually cooled at room temperature for $30 \mathrm{~min}$. Then, $1.27-\mathrm{cm}$ diameter cores were removed from each meat steak parallel to the muscle fiber. WBSF values were measured with a Warner-Bratzler shear force attachment on an Instron Universal Testing machine (Instron Corporation, Canton, MA, USA). The operating 
parameters: load cell, $50 \mathrm{~kg}$ and crosshead speed, $200 \mathrm{~mm} / \mathrm{min}$. The summary statistics for the WBSFs of the two muscles are described in Table 1.

Table 1. Summary statistics of Warner-Bratzler shear force (WBSF), intra-muscular fat (IMF), and age at slaughter in Longissimus thoracis and Semimembranosus muscle of Hanwoo.

\begin{tabular}{ccccccccc}
\hline \multirow{2}{*}{ Traits } & \multicolumn{1}{c}{ Longissimus thoracis $(\mathbf{n}=\mathbf{1 0 0 0})$} & \multicolumn{4}{c}{ Semimembranosus $(\mathbf{n}=1000)$} \\
\cline { 2 - 9 } & Mean & SD & Min & Max & Mean & SD & Min & Max \\
\hline WBSF (kg) & 3.97 & 0.86 & 2.15 & 6.92 & 6.65 & 1.14 & 3.4 & 9.88 \\
IMF (\%) & 15.83 & 4.26 & 4.82 & 29.34 & 4.89 & 1.85 & 1.5 & 15.61 \\
Age (months) & 30.8 & 1.45 & 21.2 & 36.6 & & & & \\
\hline
\end{tabular}

\subsection{Genotyping}

Three SNPs in bovine CAST and four SNPs in bovine CAPN1 were genotyped using TaqMan SNP genotyping arrays designed by Applied Biosystems (LSK, Seoul, Korea) with a Bio-Rad real-time PCR [13]. Detailed information on the locations and positions of the markers is provided in Tables $\mathrm{S} 1$ and S2. The CAPN1:c.948G $>$ C, which has a high LD relationship with CAPN1:c.580T $>$ C, marker is included in commercial test panel of the Australian GeneSTAR tenderness [10]. The linkage disequilibrium (LD) and haplotype structure for four significant SNPs within the CAPN1 gene were investigated in this study. The haplotypes for the 4 significant SNPs in the CAPN1 gene were reconstructed using default parameters in PHASE and inspected by means of Heatmap plots obtained with Haploview to visualize recombination events and to define the length of haplotypes $[15,16]$.

\subsection{Statistical Analyses}

A single-marker regression analysis was used to test for associations between the SNPs and WBSF values. Markers were assumed to be in linkage disequilibrium with quantitative trait loci in close proximity, and additive effects were evaluated. Statistical analyses were performed in ASReml [17]. To test for an association between each marker and the WBSF in different muscles, the following linear mixed regression model was utilized:

$$
Y_{i j k l m}=\mu+C G_{i}+b_{1} S_{a g e} e_{j}+b_{2} I M F_{k}+\sum_{l=1}^{7} S N P_{l}+a_{m}+e_{i j k l m}
$$

where $Y_{i j k l m}$ is the WBSF in different muscles, $\mu$ is a vector of the overall mean, $C G_{i}$ is a vector of the contemporary group for birth year, season, slaughter year, and birth place, $b_{1}$ is a regression coefficient, $\mathrm{Sage}_{j}$ is a vector of the age of slaughter as a covariate effect, $b_{2}$ is a vector of Intramuscular fat (IMF) contents as a covariate, $S N P_{l}$ is a single-locus SNP genotype coded as 0,1 , and 2 as a covariate, $a_{m}$ is a vector of random polygenic effect $\sim N\left(0, A \sigma_{a}^{2}\right)$; and $e_{i j k l m}$ is a vector of random residual $\sim N\left(0, I \sigma_{e}^{2}\right)$. The same statistical model was used for a haplotype association analysis. The percentage of the genetic variation explained by each significant SNP was calculated using the following formula [18].

$$
\% V g_{i}=100 \times \frac{2 p_{i} q_{i} a_{i}^{2}}{\sigma_{g}^{2}}
$$

where $p_{i}$ and $q_{i}$ are the allele frequencies for the ith SNP estimated for the Hanwoo population, $a_{i}^{2}$ is the estimated additive effect of the ith SNP on the WBSF phenotypes at different muscle (LT and SM), and $\sigma_{g}^{2}$ is the REML estimate of the polygenic variance in WBSF at different muscle (LT and SM) in the commercial population. 


\section{Results and Discussion}

The genotype and allele frequencies of selected seven SNPs from CAPN1 and CAST genes are presented in Table S3. The estimated allele frequencies for each marker of this study differed largely with the previous findings of [13].

This dissimilarity might be due to the three times larger sample size included in the present study than previous study. We identified a strong association between the SNP markers and WBSFs in the two muscles; the results are summarized in Table 2. Interestingly, significant effects of CAST:c.1985G $>$ C, CAPN1:c.580T>C and CAPN1:c.658T >C $(p<0.05)$ on the WBSF of both muscle types were observed. SNP variants of CAPN1 have been reported to be associated with $B$. taurus-oriented crossbred populations $[18,19]$ and a diverse genetic background consisting of $B$. taurus, $B$. indicus, and their reciprocal crossbreds $[6,20]$. These findings support our results, despite the variation in the SNP location observed across cattle populations. For example, the significantly associated CAPN1:c.948G>C SNP in the LT of B. taurus cattle [20] demonstrated insignificant associations in Hanwoo cattle that exhibited breed or population-specific SNP associations for this gene. In addition, the lack of association for all SNPs identified in our previous study of Hanwoo cattle [13] compared to our present results might be due to differences in the allele frequencies and sources of investigated samples (Table S3). In this study, we found CAPN1 and CAST gene mutation to have significant effects on the WBSF measured in both muscles of Hanwoo, as shown in the previous study [6-8]. These findings may be one of the strong evidences that CAPN1 and CAST gene mutations are strongly associated with WBSF across breeds.

Additive effects of the $C$ allele of CAST:c.1985G $>C$ and CAPN1:c.580T $>C$ were associated with an increase of 0.15 and $0.16 \mathrm{~kg}$ WBSF, and of 0.26 and $0.08 \mathrm{~kg}$ WBSF in the LT and SM, respectively. Conversely, CAPN1:c.658T>C exhibited a negative effect on the WBSF in both muscle types (Table 2). The other CAPN1:c.948G>C SNP marker exhibited insignificant effects on WBSF, despite its strong linkage disequilibrium with the CAPN1:c.580T $>C$ marker, which is the most significant marker associated with WBSFs in the LT and SM (Figure 1A). CAPN1:c.948G >C was referred to as CAPN316, which has been observed to be associated with WBSF in several studies [5,11,19].

In our study, twelve possible haplotypes were reconstructed for association analyses of CAPN1 polymorphisms with WBSF; the results are presented in Figure 1B. Haplotype TTCT had the highest frequency (27\%), followed by CTGT (19\%), TCGT (15\%), and CTGC (12\%), while the other eight haplotypes (TCCC, TTGC, TTCC, TCGC, TTGT, CCGC, TCCT, and CCGT) had a frequency of $2 \%$ to $6 \%$. Among them, six haplotypes had significant effects $(p<0.05)$ on WBSF. The second most frequent haplotype, CTGT (19\%), increased the WBSF to $0.09(p<0.01)$ and $0.10 \mathrm{~kg}(p<0.05)$ in the LT and SM, respectively (Figure 1C). However, haplotype TCGT (15\% frequency) exhibited negative effects on WBSF to $-0.09(p<0.01)$ and $-0.12(p<0.05)$. These results correspond to the single marker analysis results that CAPN1:c.580T $>C$ had a positive effect on WBSF and CAPN1:c.658T $>C$ had a negative effect. Haplotype TCGC only showed significant effects on the WBSF in the LT, while three other haplotypes (CTGC, TCCC, and TCCT) had significant effects on WBSFs in the SM. The most significant effect $(p<0.001)$ was observed for haplotype TCCT, which decreased the WBSF in the SM to $-0.39 \mathrm{~kg}$. While two variants (CAPN1:c.580T>C,CAPN1:c.658T $>C$ ) exhibited significant effects on both muscle types, the affected muscle types seem to have changed depending on the haplotype structures. Interestingly, haplotype TCCC observed at a low frequency (2\%) in the Hanwoo population had a beneficial effect $(+0.35 \mathrm{~kg})$ on the SM. The haplotype structure could change the effect of a single marker because the results were the opposite of the single marker analysis. The most frequent haplotype (TTCT) had insignificant effects on WBSF. In the future, the significant haplotype associations identified in our study can be utilized to resolve the issue reported in a previous study [13], in which no significant haplotype associations for CAPN1 in Hanwoo cattle were observed. The haplotype analysis provided more explicit results compared to a single SNP found in a particular region of the genome. (Barendse, 2011) reported that the haplotypes accounted for $80 \%$ more phenotypic variance than individual SNP in ADIPOQ, CAPN1, CXCR4, CEBPA and FASN; this evidence of a robust association with intramuscular fat content, compared to single SNP analyses of B. taurus [21]. 
Table 2. Association of CAST/CAPN1 SNP alleles on WBSF measurements for two different muscle cuts.

\begin{tabular}{|c|c|c|c|c|c|c|c|c|c|c|}
\hline \multirow{2}{*}{ Markers } & \multirow{2}{*}{ Allele } & \multirow{2}{*}{ MAF $^{a}$} & \multicolumn{4}{|c|}{ WBSF (kg) in Longissimus thoracis at 7 Days } & \multicolumn{4}{|c|}{ WBSF (kg) in Semimembranosus at 7 Days } \\
\hline & & & Effects & F-Value & $p$-Value & $\%$ Var & Effects & F-Value & $p$-Value & $\% \operatorname{Var}$ \\
\hline CAST:c.182G>A (rs109727850) & A & 0.41 & $-0.02 \pm 0.04$ & 0.33 & 0.561 & 0.03 & $-0.03 \pm 0.05$ & 0.22 & 0.631 & 0.04 \\
\hline CAST:c.1526A > G (rs109384915) & G & 0.41 & $-0.06 \pm 0.05$ & 1.91 & 0.170 & 0.28 & $-0.04 \pm 0.06$ & 1.31 & 0.255 & 0.08 \\
\hline CAST:c.1985G>C (rs110914810) & $\mathrm{C}$ & 0.08 & $0.15 \pm 0.07$ & 4.70 & $0.032 *$ & 0.53 & $0.26 \pm 0.09$ & 8.57 & $0.004^{* *}$ & 0.99 \\
\hline CAPN1:c.580T >C (rs17872079) & $\mathrm{C}$ & 0.40 & $0.16 \pm 0.06$ & 28.32 & $1.33 \times 10^{-7 * *}$ & 1.98 & $0.08 \pm 0.07$ & 10.71 & $0.001^{* *}$ & 0.31 \\
\hline CAPN1:c.658T>C (rs17872093) & $\mathrm{C}$ & 0.34 & $-0.09 \pm 0.06$ & 9.57 & $0.002^{* *}$ & 0.59 & $-0.13 \pm 0.07$ & 10.31 & $0.001^{* *}$ & 0.76 \\
\hline CAPN1:c.948G>C (rs17872000) & $\mathrm{C}$ & 0.36 & $-0.03 \pm 0.06$ & 0.43 & 0.508 & 0.07 & $-0.05 \pm 0.07$ & 0.31 & 0.574 & 0.12 \\
\hline CAPN1:c.1589T>C (rs17871051) & $\mathrm{C}$ & 0.26 & $0.03 \pm 0.04$ & 0.65 & 0.420 & 0.06 & $0.07 \pm 0.05$ & 2.11 & 0.149 & 0.19 \\
\hline
\end{tabular}

${ }^{a}$ MAF indicates a minor allele frequency; $p$-value: $0<* * 0.01<*<0.05$. 
In some previous studies, no CAPN1-CAST markers were found to be associated with the WBSFs and causal effects in different populations $[4,6,22,23]$. During the validation phase, marker effectiveness may not be equal across populations, despite commercialization of the used markers. An unmatched validation study could result from SNP frequency variation, differences in marker-causative mutation linkage phases, genetic-by-nature interactions or epistasis, as well as sample size effects and the trait measurement method [22]. Above all, single-marker associations have been utilized in various livestock studies and have commercial impact on the improvement of meat quality traits. The significantly associated SNPs found in our study could be utilized for genetic improvement of the tenderness trait in Hanwoo populations, and thereby assist in the establishment of a beef palatability prediction system in Korea.

A)

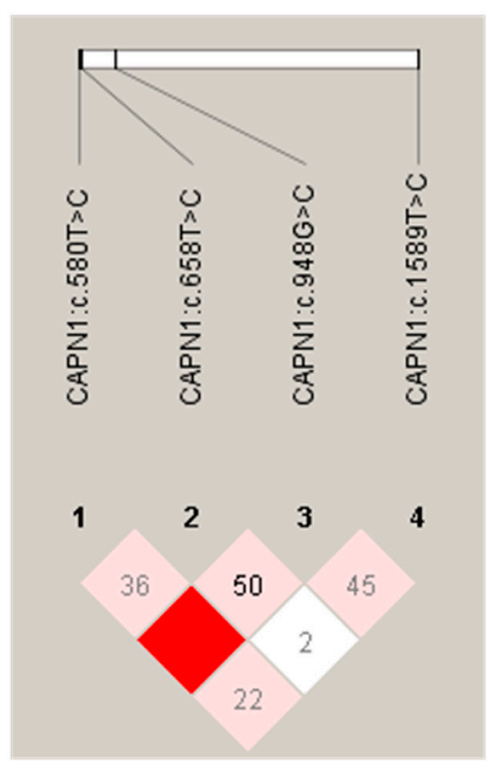

B)

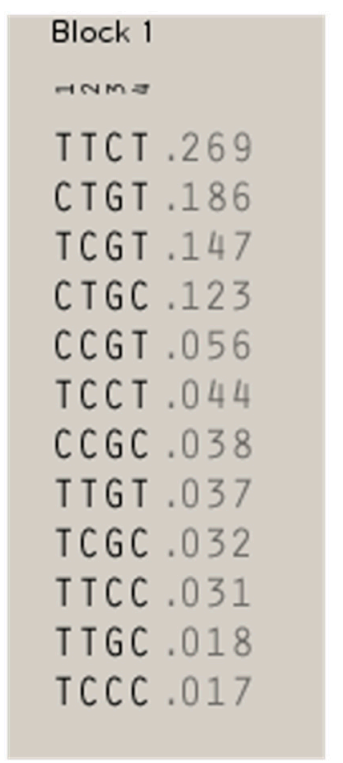

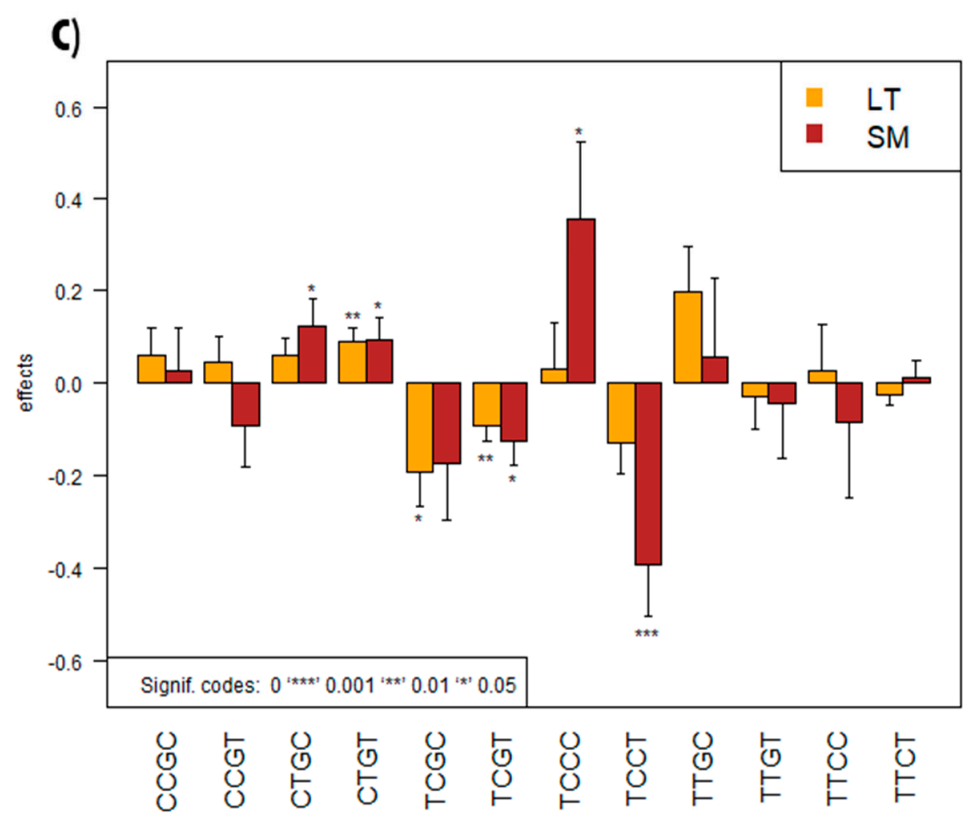

Figure 1. (A) Linkage disequilibrium pattern; (B) haplotype frequencies among the 4 SNPs in CAPN1; (C) haplotype effects on WBSF in different muscles in Hanwoo; The numbers at the top of panel (B) are equal to the numbers at panel (A) and those numbers represent each marker above. 


\section{Conclusions}

The present independent validation study confirmed the association of CAPN1 and CAST with WBSF in LT and SM muscles from Korean Hanwoo cattle. The information of significantly associated SNPs and the resulted haplotypes could be utilized in Hanwoo breeding program for further genetic improvement of tenderness traits. For the confirmed associations, additional studies are encouraged to utilize these SNPs in marker-assisted selection programs.

Supplementary Materials: The following are available online at http://www.mdpi.com/2076-2615/9/9/691/s1. Table S1, Information on the genotyped SNPs, Table S2, Sequences of the Taqman probes used for SNP genotyping, Table S3, Genotype and allele frequencies for 7 selected SNPs in CAPN1-CAST Genes.

Author Contributions: Conceptualization, S.H.L. (Seung Hwan Lee), M.H.H.; Methodology, H.-J.K., M.S.A.B.; Software, H.J.L., D.H.L.; Formal Analysis, S.H.L. (Soo Hyun Lee), S.B.J.; Data curation, S.J.; Writing-original draft, H.J.L.; Writing-review \& editing, S.H.L. (Seung Hwan Lee).

Funding: This study was funded by awards from the Molecular Breeding program (Grant no. PJ01316903) of the Next Generation BIOGREEN21 project of the National Institute of Animal Science, RDA, Republic of Korea.

Acknowledgments: All the data-set (Phenotypes and genotypes for WBSH in two muscle parties) used in this study was provided by Hanwoo Research Institutes of National Institute of Animal Science, RDA. This study was preformed to develop new genomic selection model for other meat quality traits such as WBSH. We acknowledgement to Hanwoo Research Institute of National Institute of Animal Science, RDA.

Conflicts of Interest: The authors declare that they have no competing interests.

\section{References}

1. Shackelford, S.D.; Wheeler, T.L.; Meade, M.K.; Reagan, J.O.; Byrnes, B.L.; Koohmaraie, M. Consumer impressions of Tender Select beef. J. Anim. Sci. 2001, 79, 2605-2614. [CrossRef] [PubMed]

2. Aberle, E.D.; Forrest, J.C.; Gerrard, D.E.; Mills, E.W. Principles of Meat Science, 4th ed.; Kendal/Hunt Publishing: Dubuque, IA, USA, 2001.

3. Cho, S.H.; Kim, J.; Park, B.Y.; Seong, P.N.; Kim, G.H.; Jung, S.G.; Im, S.K.; Kim, D.H. Assessment of meat quality properties and development of a palatability prediction model for Korean Hanwoo steer beef. Meat Sci. 2010, 86, 236-242. [CrossRef] [PubMed]

4. Van Eenennaam, A.L.; Li, J.; Thallman, R.M.; Quaas, R.L.; Dikeman, M.E.; Gill, C.A.; Franke, D.E.; Thomas, M.G. Validation of commercial DNA tests for quantitative beef quality traits. J. Anim. Sci. 2007, 85, 891-900. [CrossRef] [PubMed]

5. White, S.N.; Casas, E.; Wheeler, T.L.; Shackelford, S.D.; Koohmaraie, M.; Riley, D.G.; Chase, C.C., Jr.; Johnson, D.D.; Keele, J.W.; Smith, T.P.L. A new single nucleotide polymorphism in CAPN1 extends the current tenderness marker test to include cattle of Bos indicus, Bos taurus, and crossbred descent. J. Anim. Sci. 2005, 83, 2001-2008. [CrossRef] [PubMed]

6. Casas, E.; White, S.N.; Wheeler, T.L.; Shackelford, S.D.; Koohmaraie, M.; Riley, D.G.; Chase, C.C., Jr.; Johnson, D.D.; Smith, T.P.L. Effects of calpastatin and $\mu$-calpain markers in beef cattle on tenderness traits. J. Anim. Sci. 2006, 84, 520-525. [CrossRef] [PubMed]

7. Li, Y.X.; Jin, H.G.; Yan, C.G.; Seo, K.S.; Zhang, L.C.; Ren, C.Y.; Ji, X. Association of CAST gene polymorphisms with carcass and meat quality traits in Yanbian cattle of China. Mol. Biol. Rep. 2013, 40, 1875-1881. [CrossRef] [PubMed]

8. Barendse, W.J. DNA Markers for Meat Tenderness. U.S. Patent 7625698, 12 January 2009.

9. Wheeler, T.L.; Koohmaraie, M. Pre-rigor and post-rigor changes in tenderness of ovine longissimus muscle. J. Anim. Sci. 1994, 72, 32-1238. [CrossRef] [PubMed]

10. Johnston, D.J.; Graser, H.U. 2010. Estimated gene frequencies of GeneSTAR markers and their size of effects on meat tenderness, marbling, and feed efficiency in temperate and tropical beef cattle breeds across a range of production systems. J. Anim. Sci. 2010, 88, 1917-1935. [CrossRef] [PubMed]

11. Allais, S.; Journaux, L.; Leveziel, H.; Payet-Duprat, N.; Raynaud, P.; Hocquette, J.F.; Lepetit, J.; Rousset, S.; Denoyelle, C.; Bernard-Capel, C.; et al. Effects of polymorphisms in the calpastatin and $\mu$-calpain genes on meat tenderness in 3 French beef breeds. J. Anim. Sci. 2011, 89, 1-11. [CrossRef] [PubMed] 
12. Curi, R.A.; Chardulo, L.A.L.; Mason, M.C.; Arrigoni, M.D.B.; Silveira, A.C.; de Oliveira, H.N. Effect of single nucleotide polymorphisms of CAPN1 and CAST genes on meat traits in Nellore beef cattle (Bos indicus) and in their crosses with Bos taurus. Anim. Genet. 2009, 40, 456-462. [CrossRef] [PubMed]

13. Lee, S.H.; Kim, S.C.; Chai, H.H.; Cho, S.H.; Kim, H.C.; Lim, D.; Choi, B.H.; Dang, C.G.; Sharma, A.; Gondro, C.; et al. Mutations in calpastatin and $\mu$-calpain are associated with meat tenderness, flavor and juiciness in Hanwoo (Korean cattle): Molecular modeling of the effects of substitutions in the calpastatin/ $\mu$-calpain complex. Meat. Sci. 2014, 96, 1501-1508. [CrossRef] [PubMed]

14. Wheeler, T.L.; Shackelford, S.D.; Koohmaraie, M. Relationship of beef longissimus tenderness classes to tenderness of gluteus medius, semimembranosus, and biceps femoris. J. Anim. Sci. 2000, 78, 2856-2861. [CrossRef] [PubMed]

15. Stephens, M.; Smith, N.J.; Donnelly, P. A new statistical method for haplotype reconstruction from population data. Am. J. Hum. Genet. 2001, 68, 978-989. [CrossRef] [PubMed]

16. Barrett, J.C.; Fry, B.; Maller, J.; Daly, M.J. Haploview: Analysis and visualization of LD and haplotype maps. Bioinformatics 2005, 21, 263-265. [CrossRef] [PubMed]

17. Gilmour, A.R.; Gogel, B.J.; Cullis, B.R.; Thompson, R. ASReml User Guide Release 2.0; VSN International Ltd.: Hemel Hempstead, UK, 2006.

18. Falconer, D.S.; Mackay, T.F.C. Introduction to Quantitative Genetics, 4th ed.; Prentice Hall: London, UK, 1996.

19. Page, B.T.; Casas, E.; Quaas, R.L.; Thallman, R.M.; Wheeler, T.L.; Shackelford, S.D.; Koohmaraie, M.; White, S.N.; Bennett, G.L.; Keele, J.W.; et al. Association of markers in the bovine CAPN1 gene with meat tenderness in large crossbred populations that sample influential industry sires. J. Anim. Sci. 2004, 82, 3474-3481. [CrossRef] [PubMed]

20. Morris, C.A.; Cullen, N.G.; Hickey, S.M.; Dobbie, P.M.; Veenvliet, B.A.; Manley, T.R.; Pitchford, W.S.; Kruk, Z.A.; Bottema, C.D.K.; Wilson, T. Genotypic effects of calpain 1 and calpastatin on the tenderness of cooked M. longissimus dorsi steaks from Jersey $\times$ Limousin, Angus and Hereford-cross cattle. Anim. Genet. 2006, 37, 411-414. [CrossRef] [PubMed]

21. Barendse, W. Haplotype analysis improved evidence for candidate genes for intramuscular fat percentage from a genome wide association study of cattle. PLOS ONE 2011, 6, e29601. [CrossRef] [PubMed]

22. Gill, J.L.; Bishop, S.C.; McCorquodale, C.; Williams, J.L.; Wiener, P. Association of selected SNP with carcass and taste panel assessed meat quality traits in a commercial population of Aberdeen Angus-sired beef cattle. Genet. Sel. Evol. 2009, 41, 36-42. [CrossRef] [PubMed]

23. McClure, M.C.; Ramey, H.R.; Rolf, M.M.; McKay, S.D.; Decker, J.E.; Chapple, R.H.; Kim, J.W.; Taxis, T.M.; Weaber, R.L.; Schnabel, R.D.; et al. Genome-wide association analysis for quantitative trait loci influencing Warner-Bratzler shear force in five taurine cattle breeds. Anim. Genet. 2012, 43, 662-673. [CrossRef] [PubMed] 